\title{
LncRNA-AK131850 Sponges MiR-93- $5 p$ in Newborn and Mature Osteoclasts to Enhance the Secretion of Vascular Endothelial Growth Factor a Promoting Vasculogenesis of Endothelial Progenitor Cells
}

\author{
Hongyu Quan ${ }^{a}$ bengmeng Liang ${ }^{a}$ Nan Lia Ce Dou ${ }^{a}$ Chuan Liu ${ }^{a}$ Yun Bai \\ Wei Luo ${ }^{c}$ Jianmei Li $^{\mathrm{a}}$ Fei Kanga ${ }^{\mathrm{a}}$ Zhen Cao ${ }^{\mathrm{a}}$ Xiaochao Yang ${ }^{\mathrm{a}}$ Hong Jiang ${ }^{\mathrm{a}}$ \\ Shiwu Dong ${ }^{a, b}$
}

\begin{abstract}
aDepartment of Biomedical Materials Science, School of Biomedical Engineering, Third Military Medical University, Chongqing, 'b State Key Laboratory of Trauma, Burns and Combined Injury, Third Military Medical University, Chongqing, 'Department of Orthopedics, Guizhou Provincial People's Hospital, Guiyang, China
\end{abstract}

\section{Key Words}

Osteoclast • AK131850 • MiR-93-5p • VEGFa • Endothelial progenitor cells • Vasculogenesis

\begin{abstract}
Background/Aims: In the process of bone development and remodeling, the vasculature is regarded as the communicative network between the bone and neighboring tissues. Recently, it has been reported that the processes of angiogenesis and osteogenesis are coupled temporally and spatially. However, few studies reported the relationship and relevant mechanism between osteoclastogenesis and vasculogenesis. Methods: Arraystar Mouse IncRNA microarray V3.0 was firstly used to analyze the differentially expressed IncRNA genes in osteoclast different stages during osteoclastogenesis. Cell counting kit 8 (CCK-8) analysis, quantitative real-time polymerase chain reaction (qRT-PCR) analysis, migration and tube formation assays were used to detect impact of osteoclast different stages on the proliferation, differentiation, migration and tube formation of endothelial progenitor cells (EPCS), respectively. Finally, transfection of AK131850 shRNA, miR-93-5p mimic and miR-93-5p inhibitor, qRT-PCR, western blotting, enzyme-linked immunosorbent assay (ELISA), fluorescence in situ hybridization (FISH) and luciferase reporter assay were carried out to dissect molecular mechanisms. Results: In this study, we found that newborn OCs (N-OC) and mature OCs (M-OC) during osteoclastogenesis significantly promoted proliferation, differentiation, migration and tube formation of endothelial progenitor cells (EPCs). Through IncRNA microarray and GO\&pathway analysis, we found that AK131850 and co-expressed gene, vascular endothelial growth factor a (VEGFa), were significantly up-regulated in N-OC and M-OC. After inhibition of AK131850


Quan et al.: LncRNA-AK131850 of Osteoclasts Enhances Vasculogenesis

the promoting effect of $\mathrm{N}-\mathrm{OC}$ and $\mathrm{M}-\mathrm{OC}$ on EPCs was reversed. Furthermore, we found that AK131850 directly competed miR-93-5p in N-OC and M-OC through sponge, thereby increasing VEGFa transcription, expression and secretion through derepressing of miR-93-5p on VEGFa. Conclusion: Our results provided the first finding that IncRNA-AK131850 sponged miR-93-5p in N-OC and M-OC during osteoclastogenesis to enhance the secretion of VEGFa, thus promoting vasculogenesis of EPCs.

\section{Introduction}

Bone is one of the most highly vascularized and heterogeneous tissue in human body. The vasculature plays a significant role in the process of skeletal development during the embryonic stage, postnatal growth, and bone remodeling [1]. Apart from supplying the skeletal system with oxygen or nutrients and remove metabolites from the bone, the vasculature is also regarded as the communicative network between the bone and neighboring tissues [2,3]. During the skeletal development, new blood vessel formation, also termed neovascularization, can be independently achieved through angiogenesis and vasculogenesis. Angiogenesis refers to the process by which new blood vessels sprout from the existing vasculature through the recruitment of endothelial cells (ECs), while vasculogenesis refers to the de novo formation of blood vessels from endothelial progenitors circulating bone marrow-derived endothelial progenitor cells (EPCs) [4, 5]. Recently, a number of breakthrough studies have pointed out that the processes of angiogenesis and osteogenesis are coupled temporally and spatially through the communication among vascular ECs, bonebuilding osteoblasts (OBs), and bone-resorbing osteoclasts (OCs) during bone development and remodeling [6-8]. The coupling relationship between angiogenesis and osteogenesis was reported in early studies by Clemens et al., who demonstrated that HIF-1 was a key molecule that couples angiogenesis to bone formation [9]. Subsequent work by Adams et al. showed that HIF-1 increased the formation of osteoblast precursors through enhancing the new blood vessel and thus forming more independent bone formation units [10]. Besides angiogenesis, more recent data suggested that the basis for native neovascularization during postnatal development was not restricted to angiogenesis but also included vasculogenesis $[11,12]$. However, few studies investigated if and how vasculogenesis may play a role in skeletal development. Therefore, investigation of vasculogenesis in skeletal development might help to provide new information to deal with the vasculature-dependent skeletal problem which negatively affect the bone metabolism.

Bone resorption, mainly executed by OCs, is the initial event of skeletal development during the embryonic stage, postnatal growth, and bone remodeling, creating conditions for the neovascularization and bone formation [13]. At the same time, the involvement of EPCs in the early bone resorption activity is an important event for promoting skeletal development $[8,14]$. OCs are specialized multinucleated cells derived from hematopoietic stem cells or monocytes/macrophage progenitor cells [15]. There are three different stages during osteoclastogenesis: preosteoclast (Pre-OC), newborn osteoclast (N-OC) and mature osteoclast (M-OC) [16-18]. EPCs are a population of cells that are found circulating in the bone marrow and peripheral blood, which have the ability to differentiate into mature EC and then participate in vasculogenesis. There are two types of EPCs: early EPCs and late EPCs. Early EPCs show peak growth at 1 to 3 weeks and die at 4 weeks after isolation, which present a small sub-fraction from mononuclear cells, display low proliferative potential and have the ability to produce potent cytokines and growth factors. However, late EPCs appear late at 2 to 3 weeks, show exponential growth at 4 to 8 weeks and die at 12 weeks after isolation, which exclusively express endothelial cell-specific antigens, such as CD34 and Flk1 , and show extensive proliferative potential $[19,20]$. It has been reported that late EPCs produce more nitric oxide, incorporate more readily into monolayer of ECs, and form capillary tube better than early EPC [21]. In addition, many factors involved in neovascularization are released by OCs. These include not only vascular endothelial growth factor (VEGF) but 


\section{Cellular Physiology Cell Physiol Biochem 2018;46:401-417

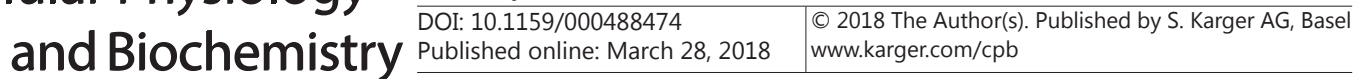

Quan et al.: LncRNA-AK131850 of Osteoclasts Enhances Vasculogenesis

also bone morphogenetic protein 7, which promotes endothelial cell survival [22], and the epithelial growth factor-like family members, that induce endothelial cell migration and tube formation [23]. Therefore, it can be believed that OCs are implicated in the involvement of neovascularization through their paracrine. Furthermore, the latest studies pointed that PreOC-releasing platelet-derived growth factor-BB (PDGF-BB) could not only induce osteoblastic differentiation of mesenchymal stem cells and promote EPCs differentiating into mature ECs in vitro, but also induce CD31 ${ }^{\text {hi }} \mathrm{Emcn}^{\text {hi }}$ vessel subtype in coupling osteogenesis in vivo [24]. This finding identified the specific involvement of OCs in the coupled vascular and osteogenic transformation pivotal to bone development and remodeling, highlighting the importance of osteoclast-derived factors in coupling bone forming activities with vascularization. From above, it's worth noting that there could be a strongly promoting effect of paracrine from OCs during osteoclastogenesis on EPCs mediated vasculogenesis. However, the effect of different stages during osteoclastogenesis on EPCs mediated vasculogenesis is still poorly studied, and the molecular mechanism remains to be elucidated.

Long non-coding RNAs (lncRNAs), that have the transcripts longer than 200 nucleotides, yet limited coding potential, are recently recognized regulating molecules involved in a variety of biological functions like gene expression, cell proliferation, and differentiation [25]. The expression profile of IncRNAs is tissue-specific and differentially expressed across various stages of cell differentiation [26]. Recently, it has been reported that lncRNAs play an important role in regulating bone development and remodeling, however, most of the functions of IncRNAs in these processes still remain unclear $[27,28]$. The regulating function of lncRNAs is not solitary but by the diverse and complex mechanism with the participation of DNA, microRNAs, mRNAs, and proteins [29-31]. Similar with lncRNAs, microRNAs, which are non-coding small RNAs composed of approximately 22 nucleotides, are also involved in controlling bone development and remodeling, including osteoclastogenesis [32-34]. In recent studies, an interesting point of view has been presented that lncRNAs are regarded as the role of sponges to make microRNAs exhausted, thus preventing mRNA from degradation by microRNA [28, 35]. In spite of these promising findings, the recognition of IncRNAs which play a vital role in different stages during osteoclastogenesis and the detailed mechanism of the genetic pathways remains to be investigated.

Based on the above studies, in this paper, we investigated whether there were lncRNAs in different stages during osteoclastogenesis regulating paracrine of these cells and thus promoting vasculogenesis mediated by EPCs. Our results provided the first demonstration that IncRNA-AK131850 sponged miR-93-5p in N-OC and M-OC during osteoclastogenesis to enhance the secretion of VEGFa, thus promoting vasculogenesis of EPCs.

\section{Materials and Methods}

\section{Cell culture}

Murine monocyte macrophage RAW264.7 cells were purchased from American Type Culture Collection (ATCC; Manassas, VA, USA). RAW264.7 cells were cultured with Dulbecco's modified Eagle's medium (DMEM; Gibco, Grand Island, NY, USA) containing 10\% fetal bovine serum (FBS; Gibco, Grand Island, NY, USA) and 1\% penicillin-streptomycin (Hyclone, Thermo Scientific, Waltham, MA, USA) at $37^{\circ} \mathrm{C}$ in a humidified atmosphere of $5 \% \mathrm{CO}_{2}$.

The EPCs were separated from the bone marrow mononuclear cells of C57BL/6 mice by density gradient centrifugation as described previously [36-38]. All experimental procedures were approved by Third Military Medical University and were performed according to the guidelines of laboratory animal care and use. Briefly, adult male C57BL/6 mice aged 8 weeks were sacrificed by cervical dislocation. Under sterilized conditions, mononuclear cells were isolated from the femora and tibia by washing the cavities with Roswell Park Memorial Institute 1640 medium (RPMI 1640; Gibco, Grand Island, NY, USA) till the femora and tibia turned white. Cell suspension was screened on a 200 mesh sieve and separated by centrifugation at centrifugal force of $400 \mathrm{~g}$ for $30 \mathrm{~min}$ by using Histopaque-1077 Hybri-Max (Sigma-Aldrich, Saint Louis, MO, USA). The middle layer containing mononuclear cells were collected and washed twice 


\section{Cellular Physiology Cell Physiol Biochem 2018;46:401-417

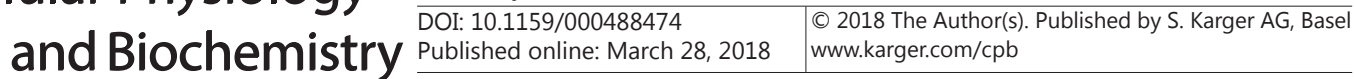

Quan et al.: LncRNA-AK131850 of Osteoclasts Enhances Vasculogenesis

with phosphate-buffered saline (PBS; Beijing Solarbio Science \& Technology, Beijing, China). After washing, cells were plated on culture dishes at the density of $5 \times 10^{6}$ and cultured with Microvascular Endothelial Cell Growth Medium-2 (EGM-2 MV) BulletKit (Lonza, Walkersville, MD, USA) in a humidified atmosphere of 5\% $\mathrm{CO} 2$ at $37^{\circ} \mathrm{C}$. Two days later, non-adherent cells were removed by washing with PBS twice and the culture medium was replaced every $3 \mathrm{~d}$. After culturing for $1 \mathrm{~d}, 7 \mathrm{~d}, 14 \mathrm{~d}$ and $28 \mathrm{~d}$, EPCs were identified by optical microscope observation, immunofluorescence for both Dil-Acetylated Low Density Lipoprotein (Dil-AcLDL; MaoKang Biotechnology, Shanghai, China) and FITC labeled Ulex Europaeus Agglutinin 1 (FITC-UEA-1; MaoKang Biotechnology, Shanghai, China), and flow cytometry (FACSCalibur Flow Cytometry, BD, Triangle, NC, USA) for PE-Cy7 conjugated anti-mouse CD34 monoclonal antibody (eBioscience, San Diego, CA) and APC conjugated anti-mouse Flk-1 monoclonal antibody (eBioscience, San Diego, CA), respectively (for all online suppl. material, see www.karger.com/doi/10.1159/000488474, Suppl. Fig.1).

\section{Induction of osteoclast differentiation at different stages}

The procedure of inducing osteoclast different stages during osteoclastogenesis was admitted and described in previous study [16, 39, 40]. In brief, RAW264.7 cells before 3 passages were cultured with osteoclast differentiation medium consisting of DMEM supplemented with $10 \%$ FBS, $1 \%$ penicillinstreptomycin, $100 \mathrm{ng} / \mathrm{ml}$ receptor activator of nuclear factor $\kappa B$ ligand (RANKL; R\&D Systems, Minneapolis, MN, USA) and $50 \mathrm{ng} / \mathrm{ml}$ macrophage colony stimulating factor (M-CSF; R\&D Systems, Minneapolis, MN, USA) for $24 \mathrm{~h}, 72 \mathrm{~h}$ and $96 \mathrm{~h}$ to gain Pre-OC, N-OC and M-OC, respectively. The RAW264.7 cells without the treatment of osteoclast differentiation medium were regarded as the Control. OCs at different stages were evaluated by tartrate-resistant acid phosphatase (TRAP) staining for differentiation, focal adhesion (FAK) staining for cytoskeleton, and quantitative real time polymerase chain reaction (qRT-PCR) for the gene expression related to differentiation and fusion (see online suppl. material, Suppl. Fig. 2). Furthermore, total RNAs from different stages were extracted by Trizol reagent (Takara, Nojihigashi, Japan) and stored at $-80^{\circ} \mathrm{C}$. Arraystar Mouse LncRNA Microarray V3.0 was performed to detect lncRNA expression by KangChen Bio-tech (Shanghai, China) and GO\&pathway analysis was used through DAVID bioinformatic resources 6.7 (http://david. abcc.ncifcrf.gov) and KEGG Pathway Database (http://www.genome.jp/kegg/pathway.html) to predict potential IncRNA function and annotation of co-expressed genes, finally qRT-PCR was carried out to verify the expression of the selected lncRNA and its underlying targets. For enzyme-linked immunosorbent assay (ELISA) of VEGFa, the supernatants from different stages were directly harvested and centrifuged at $1000 \mathrm{rpm}$ for $5 \mathrm{~min}$, and then supernatants were collected and stored at $-80^{\circ} \mathrm{C}$. For preparation of condition medium, the medium was replaced with DMEM containing 10\% FBS and 1\% penicillin-streptomycin after finishing the induction of OCs at different stages and cells were cultured at $37^{\circ} \mathrm{C}$ in a humidified atmosphere of $5 \% \mathrm{CO}_{2}$ for $48 \mathrm{~h}$. After $48 \mathrm{~h}$, the supernatants were harvested and centrifuged at $1000 \mathrm{rpm}$ for $5 \mathrm{~min}$. After centrifugation, supernatants were collected and stored at $-80^{\circ} \mathrm{C}$ for further use.

\section{Cell transfection}

For analysis of AK131850, short hairpin RNA (shRNA) against AK131850 and negative control shRNA were synthesized and constructed in the hU6-MCS-CMV-GFP-SV40-Neomycin plasmid by Shanghai Genechem Co.,Ltd (Shanghai, China). The sequences for AK131850 shRNA were as follows: 5'CGCGACCCGGAGCAAACTTCT-3'. For analysis of miR-93-5p, the miR-93-5p mimic, miR-93-5p inhibitor and the corresponding negative control were designed and synthesized by GenePharma Co., Ltd. (Shanghai, China). The sequences of miR-93-5p mimic and miR-93-5p inhibitor were: 5'-CAAAGUGCUGUUCGUGCAGGUAG-3' and 5'-CUACCUGCACGAACAGCACUUUG-3', respectively.

Transfections were performed using the Lipofectamine 2000 kit (Invitrogen, Carlsbad, USA) and Opti-MEM ${ }^{\circledR}$ I reduced serum medium (Gibco, Grand Island, NY, USA) according to the manufacturer's instructions. In brief, RAW264.7 cells before 3 passages $\left(5 \times 10^{4}\right)$ were seeded onto six-well plates and induced with osteoclast differentiation medium for $24 \mathrm{~h}, 72 \mathrm{~h}$ and $96 \mathrm{~h}$ to get Pre-OC, N-OC and M-OC, respectively. The RAW264.7 cells without the treatment of osteoclast differentiation medium were regarded as the Control. For analysis of AK131850, Control, Pre-OC, N-OC and M-OC were transfected with AK131850 shRNA (referred as to sh) and negative control shRNA (referred as to nc), respectively. For analysis of miR93-5p, N-OC and M-OC were transfected with AK131850 shRNA (referred as to sh), AK131850 shRNA and miR-93-5p mimic (referred as to sh+mimic), and AK131850 shRNA and miR-93-5p inhibitor (referred as to sh+inhibitor), respectively. The cells without transfection were used as the control (referred as to control). 
Quan et al.: LncRNA-AK131850 of Osteoclasts Enhances Vasculogenesis

After transfected for $8 \mathrm{~h}$, the medium was replaced with DMEM containing 10\% FBS and 1\% penicillinstreptomycin and cells were cultured at $37^{\circ} \mathrm{C}$ in a humidified atmosphere of $5 \% \mathrm{CO}_{2}$ for $48 \mathrm{~h}$. After $48 \mathrm{~h}$, the supernatants from different groups were harvested and centrifuged at $1000 \mathrm{rpm}$ for $5 \mathrm{~min}$, and then supernatants were collected and stored at $-80^{\circ} \mathrm{C}$ for preparation of condition medium and ELISA of VEGFa. Moreover, total RNAs from different groups were extracted using Trizol reagent and stored at $-80^{\circ} \mathrm{C}$ for qRTPCR of AK131850 and VEGFa, respectively. Total proteins from different groups were lysed in radio immune precipitation assay (RIPA) buffer (Beyotime Biotechnology, Nantong, JiangSu, China) for Western Blotting of VEGFa, respectively.

\section{Treatment of EPCs with condition medium}

Considering growth and proliferative potential, and the ability to form capillary tube, $28 \mathrm{~d}$ EPCs were chose in this study. In brief, after separated from the bone marrows and cultured for $28 \mathrm{~d}$, EPCs $\left(5 \times 10^{4}\right)$ were seeded onto six-well plates and cultured with EGM-2MV medium with equal volume of condition medium (total volume was $2 \mathrm{ml} /$ well) from above different groups. The culture medium was refreshed every $3 \mathrm{~d}$. After EPCs cultured with condition medium from Control, Pre-OC, N-OC and M-OC for $1 \mathrm{~d}, 7 \mathrm{~d}$ and $14 \mathrm{~d}$ (EGM-2MV medium was used as the blank control and referred as Blank), Cell Counting Kit-8 (CCK8) was used to detect the proliferation, qRT-PCR was applied to exam the differentiation gene expression of vascular endothelial growth factor receptor 2 (VEGFR-2), cluster of differentiation 31 (CD31), von Willebrand factor (vWF) and vascular endothelial cadherin (VE-Cadherin), migration assay was carried out to evaluate the motility and finally tube formation was conducted to observe the function of vasculogenesis, respectively. Furthermore, after EPCs cultured with condition medium of nc and sh from Control, Pre-OC, $\mathrm{N}-\mathrm{OC}$ and M-OC for $14 \mathrm{~d}$, CCK-8, qRT-PCR, migration assay and tube formation assay was carried out to evaluate the proliferation, differentiation, motility and function of vasculogenesis, respectively. Finally, after EPCs cultured with condition medium of control, sh, sh+mimic and sh+inhibitor from N-OC and M-OC for 14 d, CCK-8, qRT-PCR, migration assay and tube formation assay was carried out to evaluate the proliferation, differentiation, motility and function of vasculogenesis, respectively.

\section{CCK-8 assay}

To evaluate EPCs proliferation, the CCK-8 kit (Dojindo, Kumamoto, Japan) was used according to the manufacturers' instructions. Briefly, the medium was firstly removed from the culture plate, and then all cells were rinsed with PBS solution for 3 times. Next, cells were incubated with a mixture solution of fresh medium of EGM-2MV and CCK-8 reagent ( $/ \mathrm{v}=10: 1)$. After incubation for $3 \mathrm{~h}$, the mixture solution was added to the 96-well plate and detected with the multi-detection microplate reader (BioTek Instruments, Winooski, VT, USA) at wavelength of $450 \mathrm{~nm}$. Wells containing the CCK-8 reagent but no cells were used as the blank control.

\section{RNA extraction and $q R T-P C R$}

The extractions from each sample were stored for $15 \mathrm{~min}$ at room temperature, and treated with 200 $\mu \mathrm{l}$ of trichloromethane (Chengdu Kelong Chemical CO., LTD., Chengdu, China). After centrifuging at $4^{\circ} \mathrm{C}$ at $12000 \mathrm{rpm}$ for $15 \mathrm{~min}$, the upper phase was treated with an equal volume ( $400 \mu \mathrm{l}$ ) of isopropanol (Chengdu Kelong Chemical CO., LTD, Chengdu, China) at $-20^{\circ} \mathrm{C}$ for $2 \mathrm{~h}$ and then centrifuged at $4^{\circ} \mathrm{C}$ at $12000 \mathrm{rpm}$ for 15 min to form a pellet. Subsequently, the pellet was washed with $75 \%$ ethanol and air-dried. The isolated total RNA was then re-dissolved in $20 \mu$ diethylpyrocarbonate-treated (DEPC-treated) water (Shanghai Sangon Biology Technology and Services CO., LTD., Shanghai, China). Concentrations of the RNA samples were measured by $\mathrm{OD}_{260}$ using the ultra-low volume spectrometer (BioDrop $\mu$ Lite, Cambridge, England), and the purity was determined by the high ratio of $\mathrm{OD}_{260}$ to $\mathrm{OD}_{280}$. The reverse transcription of first strand cDNA was performed with a PrimeScript ${ }^{\mathrm{TM}} \mathrm{RT}$ reagent kit (Takara, Nojihigashi, Japan) according to the manufacturer's instructions. The qRT-PCR was performed on a CFX96 Real-Time System instrument (Bio-Rad, Hercules, CA, USA) using SYBR Premix Ex Taq II (Takara, Nojihigashi, Japan) as fluorescent probe. The cycling conditions were as follows: $95^{\circ} \mathrm{C}$ for $30 \mathrm{~s}, 40$ cycles of $95^{\circ} \mathrm{C}$ for $5 \mathrm{~s}$, and $60^{\circ} \mathrm{C}$ for $30 \mathrm{~s}$. All reactions were run in triplicate and were normalized to the reference gene $\beta$-actin. All related primers were designed and synthesized by Shanghai Sangon Biology Technology and Services CO., LTD. (Shanghai, China) and sequences were displayed in Table 1 (the primer sequences related to differentiation and fusion of OCs were shown (see online suppl. material) in Suppl. Table 1).

\section{KARGER}




\section{Cellular Physiology Cell Physiol Biochem 2018;46:401-417 \begin{tabular}{ll|l} 
and Biochemistry Published onlıne: March 28, 2018 & $\begin{array}{l}\text { (C) } 2018 \text { The Author(s). Published by S. Karger AG, Basel } \\
\text { www.karger.com/cpb }\end{array}$ \\
\hline
\end{tabular}}

Quan et al.: LncRNA-AK131850 of Osteoclasts Enhances Vasculogenesis

Migration assay

Cell migration was measured by using transwell chambers $(8-\mu \mathrm{m}$ pore size; Corning Co., Corning, NY, USA). Briefly, EPCs were re-suspended in 0 ti-MEM ${ }^{\circledR}$ I reduced serum medium and $2 \times 10^{4}$ EPCs were seeded on the upper chamber of 24well with the volume of $200 \mu \mathrm{l}$. At the same time, $600 \mu \mathrm{l}$ of EGM-2MV medium was added to the lower chamber. After $24 \mathrm{~h}$ of incubation at $37^{\circ} \mathrm{C}$ in a humidified atmosphere of $5 \% \mathrm{CO}_{2}$, cells were fixed in $4 \%$ paraformaldehyde (Boster Biological Technology, Wuhan, Hubei, China) and non-traversed cells remaining in the upper membrane were scraped off cotton swab. Traversed cells in the lower side of the membrane were stained with $0.1 \%$ crystal violet (Shanghai Sangon Biology Technology and Services CO., LTD., Shanghai, China). Under optical microscope, traversed cells were counted from five randomly chosen fields at 100× magnification and the results were reported as the number of cells per field.

\section{Tube formation assay}

Cell tube formation was measured by using Matrigel synthetic basement membrane (BD Biosciences, San Jose, CA, USA). In brief, $200 \mu$ l Matrigel was plated in each well of a 24 -well plate and allowed to polymerize at $37^{\circ} \mathrm{C}$ for $30 \mathrm{~min}$. After Matrigel polymerization, $1 \times 10^{5}$ EPCs were seeded on top of the Matrigel with $500 \mu \mathrm{l} \mathrm{EGM-2V} \mathrm{medium.} \mathrm{After} \mathrm{incubation} \mathrm{for} 6$ hours at $37^{\circ} \mathrm{C}$, tube formation was observed in 5 randomly selected microscopic fields at 100× magnification under an inverted microscope (Leica DMI600, Wetzlar, Germany). Tube formation was quantified by measuring the number of branch points and meshes by ImageJ software (National Institutes of Health, Bethesda, MD, USA).

\section{Western blotting}

The lysates of EPCs were incubated on ice for $30 \mathrm{~min}$ and then centrifuged at 12, $000 \mathrm{rpm}$ for $10 \mathrm{~min}$ to precipitate the debris. The concentration of proteins was detected by the bicinchoninic acid (BCA) protein assay kit (Beyotime Biotechnology, Nantong, JiangSu, China) and then proteins were subjected to sodium dodecyl sulfate polyacrylamide gel electrophoresis (SDS-PAGE; Beyotime Biotechnology, Nantong, JiangSu, China) followed by transferred onto Polyvinylidene Difluoride (PVDF) membranes (Immobilon ${ }^{\mathrm{TM}}-\mathrm{PSQ}$ Membranes, Sigma-Aldrich, Saint Louis, MO, USA). After blocked in 5\% skim milk (Beyotime Biotechnology, Nantong, JiangSu, China) for $1 \mathrm{~h}$, the membranes were incubated with rabbit antibodie against VEGFa (1:500, Abcam) and $\beta$-actin (1:1000, Abcam, Hong Kong, China) overnight at $4^{\circ} \mathrm{C}$ and then with the secondary antibody (1:1000, Abcam, Hong Kong, China) for $1 \mathrm{~h}$. $\beta$-actin was used as the loading control. Immunoreactive bands were visualized in ChemiDoc XRS+ imaging system (Bio-Rad, Hercules, CA, USA).

\section{ELISA}

The quantification of VEGFa in the supernatants from each sample were determined by using the Mouse VEGFa ELISA Kit (CUSABIO Biotechnology, Wuhan, Hubei, China) according to the instructions of the manufacturer. Absorbance at $450 \mathrm{~nm}$ was detected by multi-detection microplate reader. The amount of VEGFa released in the supernatants was expressed in nanogram per milliliter (ng/ml).

\section{Fluorescence in situ hybridization (FISH)}

Subcellular localization of AK131850 and VEGFa in OCs at different stages were detected by FISH kit (Guangzhou Biosense Bioscience Co., Ltd, Guangzhou, China) according to the manufacturer's instructions. In brief, RAW264.7 cells $\left(2 \times 10^{4}\right)$ were seeded on cell slides in 24-well culture plates and cultured with osteoclast differentiation medium for $24 \mathrm{~h}, 72 \mathrm{~h}$ and $96 \mathrm{~h}$ to gain Pre-OC, N-OC and M-OC, respectively. After fishing induction, cells were fixed in $4 \%$ paraformaldehyde for $30 \mathrm{~min}$ at room temperature. After permeabilization, cells were prehybridized with hybridization solution and then incubated with both of the cy3-labeled AK131850 oligonucleotide probe and FAM-labeled VEGFa oligonucleotide probe (the 


\section{Cellular Physiology Cell Physiol Biochem 2018;46:401-417

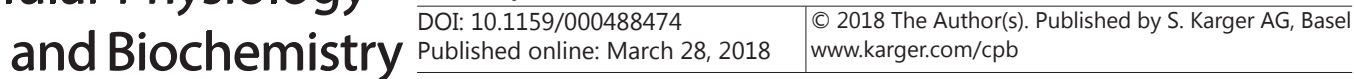

Quan et al.: LncRNA-AK131850 of Osteoclasts Enhances Vasculogenesis

sequences of probes were shown (see online suppl. material) in Suppl. Table 2). Cell nuclei were stained with 4',6-diamidino-2-phenylindole (DAPI) for $5 \mathrm{~min}$ at room temperature. Fluorescence images were obtained by laser scanning confocal microscope (LSM700, Carl Zeiss, Germany).

\section{Luciferase reporter assay}

The fragments of wild-type 3'-UTR and mutated 3'-UTR of VEGFa including the putative miR-93-5p binding sites predicted by RegRNA 2.0 (http://regrna2.mbc.nctu.edu.tw/) and full sequences of AK131850 wild-type and mutated containing miR-93-5p binding sites predicted by RegRNA 2.0 (http://regrna2. mbc.nctu.edu.tw/) and Starbase v2.0 (http://starbase.sysu.edu.cn/mirLncRNA.php) were cloned into the downstream of luciferase gene in psiCHECK ${ }^{\mathrm{TM}}$-2 luciferase reporter vector by Oligobio Co., Ltd (Beijing, China) and named as the reporter vectors VEGFa WT, VEGFa MT, AK131850 WT, and AK131850 MT, respectively (the sequences were shown, see online suppl. material, in Suppl. Table 3). For cotransfection, HEK 293T cells (ATCC; Manassas, VA, USA) were plated in 96-well plate and cotransfected with 100 ng of AK131850 WT, AK131850 MT, VEGFa WT, or VEGFa MT and $100 \mathrm{nM}$ miR-93-5p mimic or nc by using Lipofectamine 2000 and and Opti-MEM ${ }^{\circledR}$ I reduced serum medium. After transfection for $48 \mathrm{~h}$, the luciferase activities were measured by using the dual luciferase reporter assay system (Promega, Madison, WI, USA) according to the manufacturer's protocol. Renilla luciferase activities were normalized to the firefly luciferase activities and the data were expressed as the fold change relative to the corresponding control groups which were defined as 1.0 .

\section{Statistical analysis}

All data were representative of at least three experiments. The quantitative data were reported as means \pm standard deviation (SD). Statistical differences were carried out by using the unpaired Student's $t$ test between two groups and using one-way ANOVA followed by Student-Newman-Keuls post hoc test between multiple group. All statistical analyses were conducted by using the SPSS 20.0 (SPSS Inc., Chicago, IL, USA). A value of $P<0.05$ indicated statistically significant.

\section{Results}

AK131850 and its co-expressed VEGFa are significantly up-regulated in N-OC and M-OC during osteoclastogenesis

To understand potential involved lncRNAs in osteoclast different stages during osteoclastogenesis, Arraystar Mouse IncRNA microarray V3.0 that contained probe sequences for 35923 mouse lncRNAs was used to analyze the differentially expressed lncRNA genes. Our results confirmed that there were 8412 lncRNAs differentially expressed in osteoclast different stages during osteoclastogenesis and the expression profiles were presented as the cluster diagram (Fig. 1A). According to the relationship of lncRNAs and their nearby coding genes (within $300 \mathrm{~kb}$ ) which might be involved in regulating neovascularization, we found that AK131850 with the nearby gene of VEGFa was significantly up-regulated in N-OC (fold $>2, P<0.05$ ) and the relationship between AK131850 and VEGFa was natural antisense. Accordingly, both AK131850 and VEGFa were selected for further investigation. Following by microarray analysis, GO\&pathway analysis was used to predict the potential function of AK131850 and co-expressed genes. As shown in Fig. 1B, the results demonstrated that AK131850 could co-express with VEGFa, FGF-1, sFlt1, H2-M2 and Tnk1 in osteoclast different stages during osteoclastogenesis, suggesting that AK131850 and its co-expressed genes might play an important role in skeletal development. By finding and reading references, we found that VEGFa, FGF-1 and sFlt1 played a vital role in vasculature [41-44]. Therefore, qRT-PCR was used to further confirm the mRNA expression of AK131850 and co-expressed genes of VEGFa, FGF-1 and sFlt1, and results of significant data are summarized in the graphs shown in Fig. 1C. Remarkable increased level of AK131850 was observed in Pre-OC, N-OC and $\mathrm{M}-\mathrm{OC}$ compared with that in Control, and it was much higher in the $\mathrm{N}-\mathrm{OC}$ and $\mathrm{M}-\mathrm{OC}$ than that in Pre-OC. In addition, obvious increased level of VEGFa was found in Pre-OC, N-OC and $\mathrm{M}-\mathrm{OC}$, and N-OC and M-OC showed higher level of VEGFa expression than Pre-OC. However, it 


\section{Cellular Physiology \begin{tabular}{ll|l} 
and Biochemistry & Dublished online: March 28, 2018 & $\begin{array}{l}\text { (c) } 2018 \text { The Author(s). Published by S. Karger AG, Basel } \\
\text { www.karger.com/cpb }\end{array}$ \\
\hline
\end{tabular}}

Quan et al.: LncRNA-AK131850 of Osteoclasts Enhances Vasculogenesis

Fig. 1. AK131850 is significantly up-regulated with it co-expressed VEGFa in N-OC and M-OC. (A) The cluster heat map of expression profiles from 8412 lncRNAs at different stages during osteoclastogenesis measured by Arraystar Mouse IncRNA microarray V3.0. (B) The potential co-expressed genes with AK131850 predicted by GO\&pathway analysis. (C) mRNA levels of AK131850, VEGFa, FGF-1 and sFlt1 in osteoclast different stages during osteoclastogenesis assessed by qRTPCR. Data are shown as means \pm SD. ${ }^{*} \mathrm{P}<0.05$.

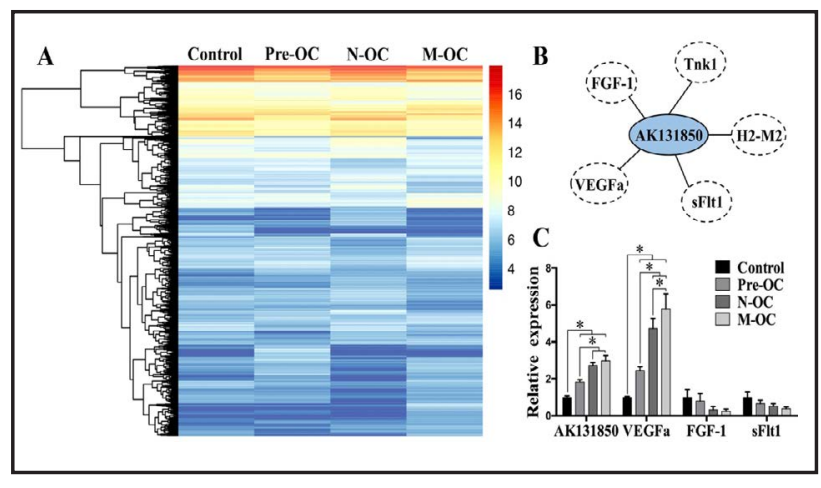

was noticed that the expression of FGF-1 and sFlt1 was not significantly changed in osteoclast different stages during osteoclastogenesis. Taken together, the results suggested that the expression of AK131850 and its coexpressed gene VEGFa were up-regulated in osteoclast different stages during osteoclastogenesis; besides, $\mathrm{N}-\mathrm{OC}$ and $\mathrm{M}-\mathrm{OC}$ showed higher mRNA expression levels of AK131850 and VEGFa than Pre-OC. Whether AK131850 and its co-expressed VEGFa in osteoclast different stages, especially in $\mathrm{N}-\mathrm{OC}$ and $\mathrm{M}-\mathrm{OC}$, were involved in skeletal development and neovascularization still remained to be elucidated. To assess this hypothesis, further experiments were performed.

$\mathrm{N}-\mathrm{OC}$ and M-OC remarkably promotes proliferation, differentiation, migration and tube formation of EPCS

To investigate the effect of osteoclast different stages during osteoclastogenesis on EPCs, condition medium from Control, Pre-OC, N-OC and M-OC was collected. Af-

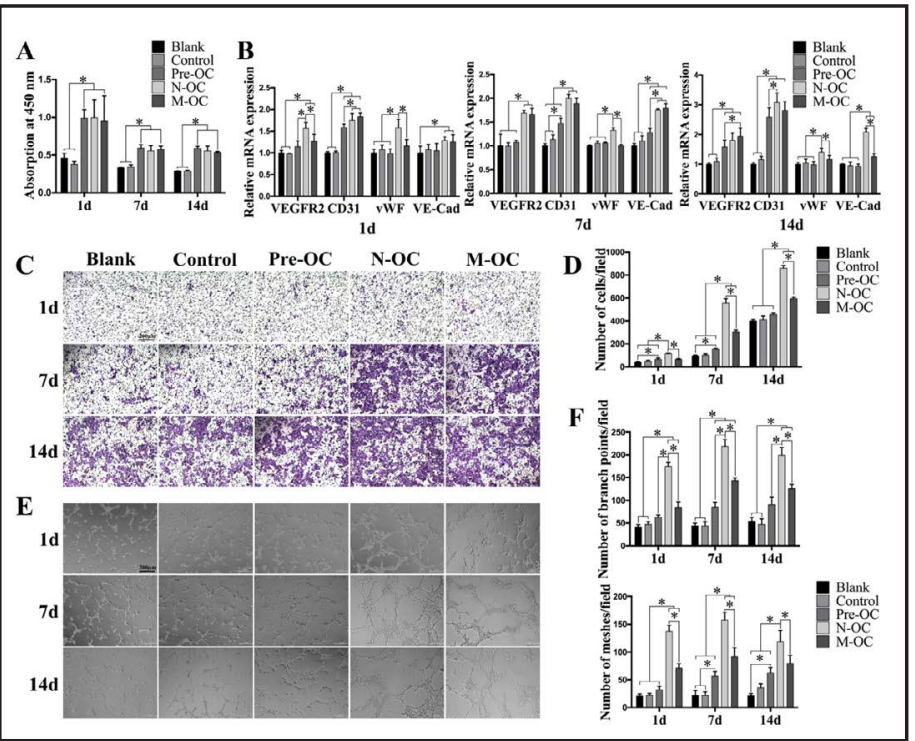

Fig. 2. N-OC and M-OC promotes proliferation, differentiation, migration and tube formation of EPCs. (A) Absorption at $450 \mathrm{~nm}$ of EPCs treated with condition medium from Control, Pre-OC, N-OC and M-OC detected by CCK-8 assay at $1 \mathrm{~d}, 7 \mathrm{~d}$ and $14 \mathrm{~d}$. EGM-2MV medium was used as the blank control. (B) mRNA expression levels of VEGFR2, CD31, vWF and VE-Cadherin in EPCs treated with condition medium from Control, Pre-OC, N-OC and M-OC detected by qRT-PCR at $1 \mathrm{~d}$, $7 \mathrm{~d}$ and $14 \mathrm{~d}$. EGM-2MV medium was used as the blank control. (C) Light microscopic images of EPCs treated with condition medium from Control, Pre- OC, N-OC and M-OC at $1 \mathrm{~d}, 7 \mathrm{~d}$ and $14 \mathrm{~d}$ assessed by migration assay. EGM-2MV medium was used as the blank control. Scale bar, $200 \mu \mathrm{m}$. (D) Number of migrated EPCs quantified by ImageJ software. (E) Inverted microscope images of EPCs treated with condition medium from Control, Pre-OC, N-OC and M-OC at $1 \mathrm{~d}, 7 \mathrm{~d}$ and $14 \mathrm{~d}$ assessed by tube formation assay. EGM-2MV medium was used as the blank control. Scale bar, $200 \mu \mathrm{m}$. (F) Number of branch points and meshes quantified by ImageJ software. Data are shown as means $\pm \mathrm{SD} .{ }^{*} \mathrm{P}<0.05$.

ter cultured with condition medium for $1 \mathrm{~d}, 7 \mathrm{~d}$, and $14 \mathrm{~d}$, CCK-8, qRT-PCR, migration and tube formation assays were used to detect the proliferation, differentiation, migration and tube formation of EPCs, respectively. CCK-8 results showed that Pre-OC, N-OC and M-OC exhibited significantly higher 
Fig. 3. Inhibition of AK131850 reverses the promoting effect of $\mathrm{N}-\mathrm{OC}$ and $\mathrm{M}-\mathrm{OC}$ on EPCs. (A) Absorption at $450 \mathrm{~nm}$ of EPCs treated with condition medium from sh and nc of Control, Pre-OC, N-OC and M-OC detected by CCK-8 assay at 14 d. (B) mRNA expression levels of VEGFR2, CD31, vWF and VE-Cadherin in EPCs treated with condition medium from sh and nc of Control, Pre-OC, N-OC and M-OC measured by qRT- PCR at 14 d. (D) Light microscopic images of EPCs treated with condition medium from sh and nc of Control, Pre-OC, N-OC and $\mathrm{M}-\mathrm{OC}$ at $14 \mathrm{~d}$ assessed by migration assay. EGM-2MV medium was used as the blank control. Scale bar, $200 \mu \mathrm{m}$. (C) Number of migrated EPCs quantified by ImageJ software. (E) Inverted microscope images of EPCs treated with condition medium from sh and nc of Control, Pre-OC, N-OC and M- OC at $14 \mathrm{~d}$ assessed by tube formation assay. EGM-2MV medium was used as the blank control. Scale bar, $200 \mu \mathrm{m}$. (F) Number of branch points and meshes quantified by ImageJ software. Data are shown as means $\pm \mathrm{SD}$. ${ }^{*} \mathrm{P}<0.05$.

absorption of EPCs at $450 \mathrm{~nm}$ than Control and Blank at $1 \mathrm{~d}, 7 \mathrm{~d}$ and $14 \mathrm{~d}$, respectively (Fig. 2A). For qRT-PCR, N-OC and M-OC could remarkably enhance the mRNA expression levels of EPC-related differentiation genes VEGFR2, CD31, vWF and VE-Cadherin at $1 \mathrm{~d}, 7 \mathrm{~d}$ and 14 d (Fig. 2B). Furthermore, the migration results showed the presence of significantly more migrating EPCs treated with condition medium from N-OC and M-OC compared with those from Blank, Control and Pre-OC at $1 \mathrm{~d}, 7 \mathrm{~d}$ and $14 \mathrm{~d}$ (Fig. 2C and 2D). As shown in Fig. 2E and $2 \mathrm{~F}$, the EPCs cultured with condition medium from N-OC and M-OC for $1 \mathrm{~d}, 7 \mathrm{~d}$ and $14 \mathrm{~d}$ exhibited large amounts of branching points and meshes, while the EPCs cultured with condition medium from Blank, Control and Pre-OC formed limited branching points and meshes. It was worth noting that $\mathrm{N}-\mathrm{OC}$ showed the highest promoting effect on EPC differentiation, migration and tube formation among other stages of OCs during osteoclastogenesis. Taken together, $\mathrm{N}-\mathrm{OC}$ and $\mathrm{M}-\mathrm{OC}$ remarkably promoted the proliferation, differentiation, migration and tube formation of EPCs, and N-OC had the highest promoting effect on EPCs. In consideration of that the promoting effect of osteoclast different stages on EPCs were obviously higher at $14 \mathrm{~d}$ than those at $1 \mathrm{~d}$ and $7 \mathrm{~d}, 14 \mathrm{~d}$ was chosen for the duration of culture period that EPCs were treated with the condition medium in the following experiments.

\section{Inhibition of AK131850 reverses the promoting effect of N-OC and M-OC on EPCs}

To explore whether AK131850 took part in regulating the promoting effect of osteoclast different stages on EPCs, Control, Pre-OC, N-OC and M-OC were transfected with shRNA against AK131850 and negative control shRNA, respectively. After $48 \mathrm{~h}$ transfection, the condition medium from different stages was collected and used to treat EPCs for $14 \mathrm{~d}$. After $14 \mathrm{~d}$ treatment, CCK-8, qRT-PCR, migration and tube formation assays were used to detect the proliferation, differentiation, migration and tube formation of EPCs, respectively. CCK8 results found that after the inhibition of AK131850 in Control, Pre-OC, N-OC and M-OC, the absorption at $450 \mathrm{~nm}$ of EPCs was significantly decreased (Fig. 3A). For qRT-PCR, remarkable decreased mRNA levels of VEGFR2, CD31, vWF and VE-Cadherin were observed in the sh of Control, Pre-OC, N-OC and M-OC in comparison to those in the nc of Control, Pre-OC, N-OC and M-OC, respectively (Fig. 3B). Moreover, the migration results exhibited the 
Fig. 4. AK131850 accelerates transcription, expression and secretion of VEGFa. (A) qRT-PCR analysis of AK131950 mRNA expression level in Control, Pre-OC, N-OC and M-OC after transfected with AK131850 shRNA. (B) qRT-PCR analysis of VEGFa mRNA expression level in Control, PreOC, N-OC and M-OC after transfected with AK131850 shRNA and negative control. (C) Protein expression level of VEGFa in
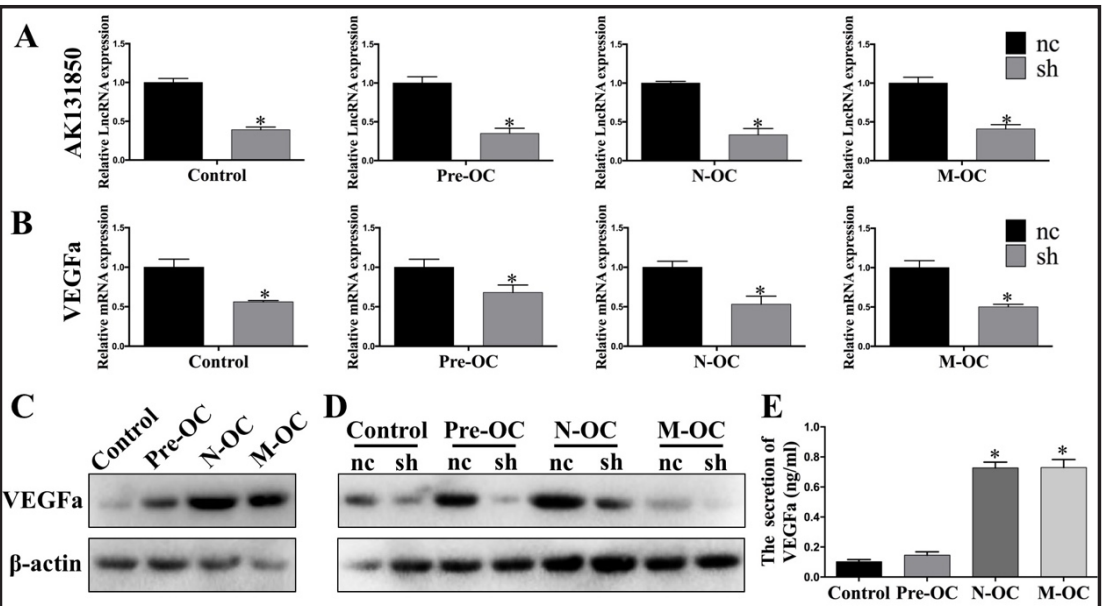

F
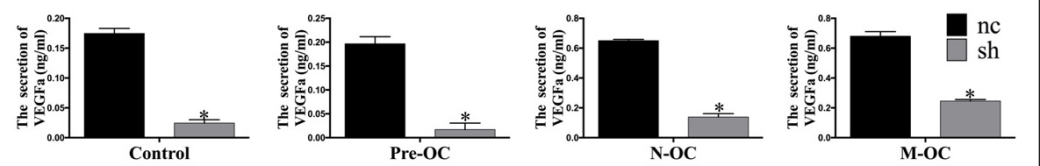

Control, Pre-OC, N-OC and M-OC measured through western blotting analysis. (D) Western blotting analysis of VEGFa protein expression level in Control, Pre-OC, N-OC and M-OC after transfected with AK131850 shRNA and negative control. (E) Secretion level of VEGFa in Control, Pre-OC, N-OC and M-OC measured by ELISA. (F) ELISA analysis of VEGFa secretion level in Control, Pre-OC, N-OC and M-OC after transfected with AK131850 shRNA and negative control. Data are shown as means $\pm \mathrm{SD}$. ${ }^{*} \mathrm{P}<0.05$.

presence of significantly fewer migrating EPCs treated with condition medium from the sh of Control, Pre-OC, N-OC and M-OC compared with those from nc of Control, Pre-OC, N-OC and M-OC, respectively (Fig. 3C and 3D). As shown in Fig. 3E and 3F, the EPCs cultured with condition medium from the sh of Control, Pre-OC, N-OC and M-OC formed small amounts of branching points and meshes, while the EPCs cultured with condition medium from the nc of Control, Pre-OC, N-OC and M-OC shaped into a large number of branching points and meshes. Taken together, above results demonstrated that inhibition of AK131850 reversed the promoting effect of osteoclast different stages on proliferation, differentiation, migration and tube formation of EPCs, validating the hypothesis that remarkably increased AK131850 in $\mathrm{N}-\mathrm{OC}$ and $\mathrm{M}-\mathrm{OC}$ during osteoclastogenesis could regulate vasculogenesis during skeletal development.

\section{AK131850 regulates gene transcription, protein expression and secretion of VEGFa}

To investigate whether AK131850 regulated its nearby co-expressed coding gene VEGFa in osteoclast different stages as a result of promoting proliferation, differentiation, migration and tube formation of EPCs, Control, Pre-OC, N-OC and M-OC were transfected with shRNA against AK131850 and negative control shRNA, respectively. After transfected for $48 \mathrm{~h}$, qRTPCR, western blotting and ELISA were carried out to exam the mRNA expression, protein expression and secretion levels of VEGFa. At the same time, the protein expression and secretion levels of VEGFa in Control, Pre-OC, N-OC and M-OC without transfection were also detected. The results of qRT-PCR demonstrated that the mRNA expression levels of AK131850 and VEGFa were significantly decreased in Control, Pre-OC, N-OC and M-OC, after transfected with AK131850 shRNA, respectively (Fig. 4A and 4B). At the protein level, the expression of VEGFa was obviously higher in N-OC and M-OC, meanwhile, slightly higher in Pre-OC when compared with that in Control (Fig. 4C). However, after transfected with AK131850 shRNA, the protein expression of VEGFa in Control, Pre-OC, N-OC and M-OC was remarkably decreased (Fig. 4D). VEGFa gene were then sent for ELISA analysis to verify if the high expression of mRNA and protein could be effectively changed into protein secretion 
Fig. 5. AK131850 upregulates VEGFa by sponging miR-93-5p in N-OC and M-OC. (A) Laser confocal microscope fluorescence images of AK131850 and VEGFa in Control, Pre-OC, N-OC and M-OC detected by FISH. Scale bar, $10 \mu \mathrm{m}$. (B) Schematic illustration of the presumed miR-93-5p binding sites and mutant sites in AK131850 and VEGFa sequences. (C) Luciferase reporter activity of HEK 293T cells after cotransfected with AK131850 WT,

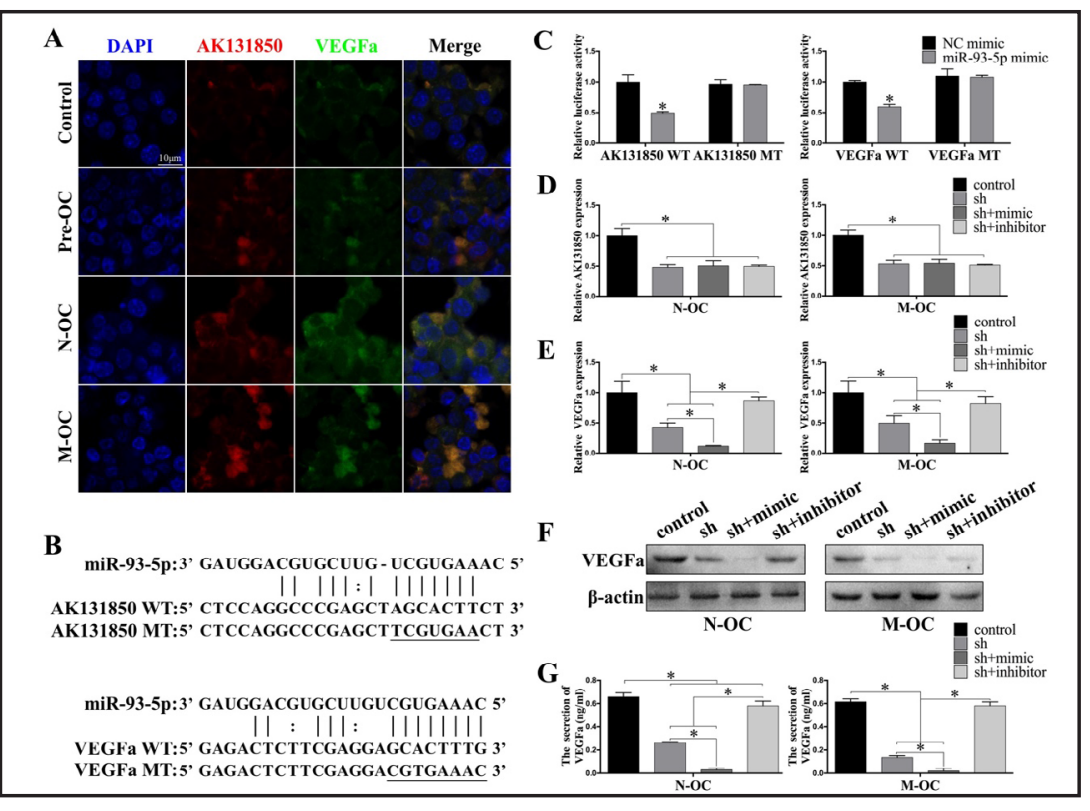
AK131850 MT, VEGFa WT, or VEGFa MT and miR-93-5p mimic or negative control. (D) qRT-PCR analysis of AK131850 mRNA expression level in N-OC and M-OC after transfected with AK131850 shRNA, miR-93-5p mimic and miR-93-5p inhibitor. The cells without transfection were used as the control. (E) qRT-PCR analysis of VEGFa mRNA expression level in N-OC and M-OC after transfected with AK131850 shRNA, miR-93-5p mimic and miR-93-5p inhibitor. (F) Western blotting analysis of VEGFa protein expression level in N-OC and M-OC after transfected with AK131850 shRNA, miR-93-5p mimic and miR-93- 5p inhibitor. (G) ELISA analysis of VEGFa secretion level in N-OC and M-OC after transfected with AK131850 shRNA, miR-93-5p mimic and miR-93-5p inhibitor. The cells without transfection were used as the control. Data are shown as means \pm SD. $*$ P $<0.05$.

and the results were shown in Fig. 4E. The protein secretion of VEGFa showed unambiguous elevation in N-OC and M-OC. Consistent with the results of qRT-PCR and western blotting, after transfected with AK131850 shRNA, the secretion of VEGFa in Control, Pre-OC, N-OC and M-OC was significantly down-regulated (Fig. 4F). To sum up, it was believed that AK131850 promoted its nearby co-expressed gene VEGFa transcription, protein expression and secretion in N-OC and M-OC. Based on the above-mentioned results, N-OC and M-OC were selected as the target cells to be used in the following investigation.

AK131850 up-regulates VEGFa by competing for miR-93-5p binding in N-OC and M-OC

To gain insights into the precise mechanism by which AK131850 modulates VEGFa, FISH was used firstly to observe the distribution of AK131850 and VEGFa in osteoclast different stages. As seen in Fig. 5A, AK131850 and VEGFa were found to be primarily localized in the cytoplasm of osteoclast different stages. This finding provided the evidence that AK131850 might act as endogenous sponge RNA to interact with the miRNA, existed only in cytoplasm, and influence the expression of the miRNA as well as its target mRNA. Accordingly, bioinformatic prediction was used by RegRNA 2.0 and Starbase v2.0 to find the miRNA that might bind to both of AK131850 and VEGFa. As expected, miR-93-5p was found to contain the potential conserved binding sites for both of AK131850 and VEGFa (Fig. 5B). For validation, luciferase reporters containing AK131850 WT or MT of the miR93-5p binding site were constructed and co-transfected with miR-93-5p into 293T cells. It could be found that the miR-93-5p mimic significantly suppressed the luciferase activities of the AK131850 WT, whereas it had less influence on the AK131850 MT or empty vector (Fig. 5C). In addition, to find out whether the binding site on miR-93-5p binds to the VEGFa 3'UTR, luciferase reporters containing the miR-93-5p binding site on the VEGFa 3'UTR 


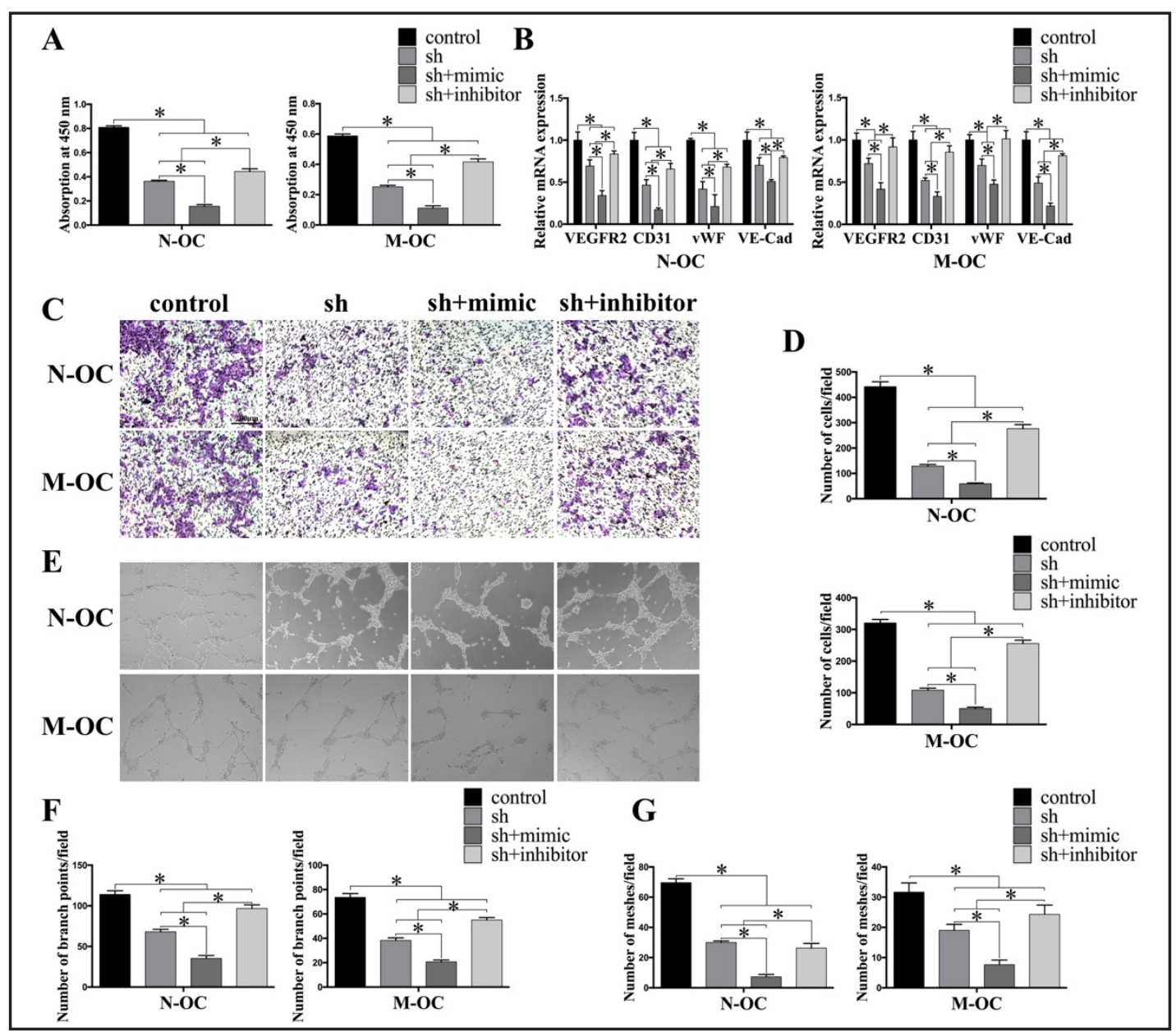

Fig. 6. AK131850 execute the promoting effect of N-OC and M-OC on EPCs through sponging miR-93-5p. (A) Absorption at $450 \mathrm{~nm}$ of EPCs treated with condition medium from sh, sh+mimic, sh+inhibitor of N-OC and M-OC detected by CCK-8 assay at $14 \mathrm{~d}$. (B) mRNA expression levels of VEGFR2, CD31, vWF and VE- Cadherin in EPCs treated with condition medium from sh, sh+mimic, sh+inhibitor of N-OC and M-OC measured by qRT-PCR at $14 \mathrm{~d}$. (C) Light microscopic images of EPCs treated with condition medium from sh, sh+mimic, sh+inhibitor of N-OC and M-OC at $14 \mathrm{~d}$ assessed by migration assay. Scale bar, $200 \mu \mathrm{m}$. (D) Number of migrated EPCs quantified by ImageJ software. (E) Inverted microscope images of EPCs treated with condition medium from sh, sh+mimic, sh+inhibitor of N-OC and M-OC at $14 \mathrm{~d}$ assessed by tube formation assay. Scale bar, 200 $\mu \mathrm{m}$. (F) Number of branch points and meshes quantified by ImageJ software. The cells without transfection were used as the control. Data are shown as means \pm SD. $* \mathrm{P}<0.05$.

was constructed. Similarly, the miR-93-5p mimic obviously down-regulated the luciferase activities of the VEGFa WT but not the VEGFa MT or empty vector (Fig. 5C). To further confirm whether AK131850 acted as endogenous sponge RNA to interact with miR-93-5p and influence the expression of its target gene VEGFa, N-OC and M-OC were transfected with AK131850 shRNA, AK131850 shRNA and miR-93-5p, and AK131850 shRNA and miR93-5p inhibitor, respectively. After transfection, qRT-PCR, western blotting and ELISA were respectively used to detect the mRNA expression, protein expression and secretion levels of VEGFa. As shown in Fig. 5D, the AK131850 mRNA expression in N-OC and M-OC were significantly decreased after transfected with AK131850 shRNA, AK131850 shRNA plus miR93-5p mimic and AK131850 shRNA plus miR-93-5p inhibitor, respectively, but there was no significant difference among them. Most importantly, the mRNA expression level of VEGFa in N-OC and M-OC were remarkably down-regulated after transfected with AK131850 shRNA and further decreases were found after cotransfected with miR-93-5p mimic, meanwhile, 


\section{Cellular Physiology Cell Physiol Biochem 2018;46:401-417 \\ \begin{tabular}{l|l} 
DOI: 10.1159/000488474 & $\begin{array}{l}\text { O 2018 The Author(s). Published by S. Karger AG, Basel } \\
\text { www.karger.com/cpb }\end{array}$
\end{tabular}}

Quan et al.: LncRNA-AK131850 of Osteoclasts Enhances Vasculogenesis

reverses were also detected after cotransfected with miR-93-5p inhibitor (Fig. 5E). At the same time, the protein expression and secretion were consistent with the mRNA expression and the results were shown in Fig. 5F and 5G, respectively. In summary, it was certain that AK131850 is highly expressed in N-OC and M-OC and it sponged miR-93-5p sponged miR93-5p, thus up-regulating the transcription, protein expression and secretion of its target gene VEGFa.

AK131850 in N-OC and M-OC promotes proliferation, differentiation, migration and tube formation of EPCs through sponging miR-93-5p

To further confirm whether the promoting effect of N-OC and M-OC on EPCs through VEGFa was modulated by the sponge of AK131850 on miR-93-5p, EPCs were treated with the condition medium from the control, sh, sh+mimic and sh+inhibitor of N-OC and M-OC for $14 \mathrm{~d}$, respectively. Fourteen days later, CCK-8, qRT-PCR, migration and tube formation assays were used to detect the proliferation, differentiation, migration and tube formation of EPCs, respectively. CCK-8 results indicated that after AK131850 suppression in N-OC and $\mathrm{M}-\mathrm{OC}$, the absorption at $450 \mathrm{~nm}$ of EPCs was significantly decreased and further decreases were found after miR-93-5p overexpression, meanwhile, obviously increases were also detected after miR-93-5p inhibition (Fig. 6A). For qRT-PCR, remarkable decreased mRNA levels of VEGFR2, CD31, vWF and VE-Cadherin were observed in the sh of N-OC and M-OC, and further reductions were observed after the overexpression of miR-93-5p, at the same time, dramaticlly increases were also detected after the inhibition of miR-93-5p (Fig. 6B). In addition, the migration results exhibited the presence of significantly fewer migrating EPCs treated with condition medium from the sh of $\mathrm{N}-\mathrm{OC}$ and $\mathrm{M}-\mathrm{OC}$, while overexpression of miR-93-5p could further decreased the number of migrated cells and inhibition of miR-93$5 p$ could reverses the down-regulations induced by AK131850 inhibition (Fig. 6C and 6D). Similarly, the EPCs cultured with condition medium from the sh of N-OC and M-OC formed fewer branching points and meshes, while overexpression of miR-93-5p could further decreased the number of branch points and meshes, and inhibition of miR-93-5p could reverses the down-regulations induced by AK131850 inhibition (Fig. 6E, 6F and 6G). To sum up, our findings suggested that AK131850 sponged miR-93-5p to enhance the secretion of VEGFa in N-OC and M-OC, thus promoting proliferation, differentiation, migration and tube formation of EPCs.

\section{Discussion}

New blood vessel formation is necessary for skeletal development during the embryonic stage, postnatal growth, and bone remodeling [6]. Recently, it has been reported that the relative abundance of the $\mathrm{H}$-type vessels is important in the processes of bone formation or bone loss [7, 10]. Additionally, Xie et al. revealed that PDGF-BB secreted by Pre-OC controlled the formation of the H-type vessel subtype to enhance the coupling of bone formation and angiogenesis, suggesting the importance of OCs in promoting vascularization during bone development and remodeling [24]. It was very interesting in our study to find that N-OC and M-OC could remarkably promote the proliferation, differentiation, migration and tube formation of EPCs, and N-OC showed the best promoting effect. Similarly, our findings also identified the specific participation of OCs in vasculogenesis during bone development and remodeling. On the basis of above findings, it could be sure that OCs at different stages during osteoclastogenesis all acted as vital roles in modulating neovascularization pivotal to bone development and remodeling.

Accumulating evidence suggests that IncRNAs is tissue-specific and differentially expressed across varying stages during cell differentiation [26, 45]. Furthermore, emerging findings from Zhu etal. have pointed out that the bone development and remodeling processes are regulated by lncRNAs [46]. They demonstrated that lncRNA ANCR expression was higher in human fetal osteoblastic cell line hFOB1.19, while was remarkably decreased during 
Fig. 7. Schematic diagram of VEGFa secreted by N-OC and M-OC to promote vasculogenesis of EPCs.

the differentiation, because lncRNA ANCR physically interacted with enhancer of zeste homolog 2, resulting in suppression of a key transcription factor Runx2 through its promoter region methylation. Interestingly, our results indicated that lncRNAs differentially expressed in osteoclast

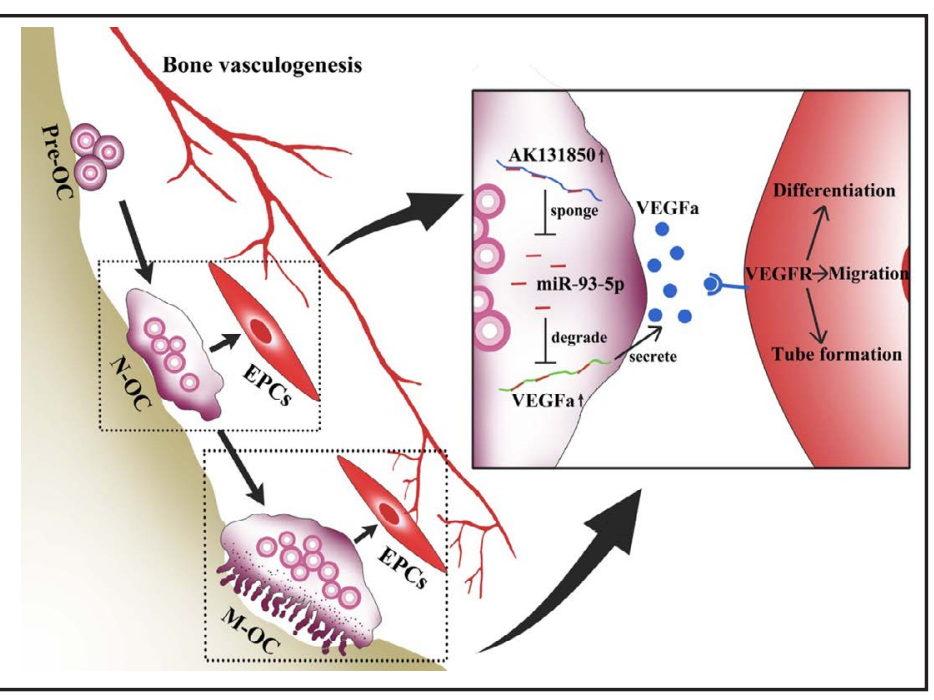
different stages during osteoclastogenesis. Importantly, the expression of AK131850 were up-regulated during osteoclastogenesis; besides, N-OC and M-OC expressed higher mRNA level of AK131850 than Pre-OC and Control. After transfected with AK131850 shRNA, the effect of OCs during osteoclastogenesis was obviously reversed, identifying the involvement of AK131850 closely related to osteoclastogenesis and vasculogenesis.

VEGF has been proven to be one of the most important modulators involved in process of neovascularization via VEGFR-mediated signaling pathways and acts as a pivotal part in bone development $[47,48]$. Evidence has shown that VEGF, is produced by most parenchymal cells and acts in a paracrine manner on adjacent vascular cells to regulate neovascularization [22]. Recently, it has been reported that transforming growth factor- $\beta$ (TGF- $\beta$ ) stimulated OBs could synthesis VEGF through the negative regulator heat shock protein 70 [49]. In vasculogenesis process, VEGF is indispensable for recruitment, retention, proliferation, differentiation, migration and capillary-like structure formation of EPCs [50, 51]. In addition, the VEGF family is a family of homodimeric proteins consisting of six members: VEGF-A, VEGF-B, VEGF-C, VEGF-D, VEGF-E and placenta growth factor [48]. VEGFa has been studied mostly of the family and is one of the main promoters of both physiological and pathological neovascularization $[52,53]$. In this study, it found that VEGFa was highly expressed in $\mathrm{N}-\mathrm{OC}$ and $\mathrm{M}-\mathrm{OC}$, of which the gene transcription, protein expression and secretion were modulated by AK131850. These findings pointed out the co-expression relationship between AK131850 and VEGFa. Recently, Fabbri et al. has predicted the binding sites of miR-93-5p in the 3'UTR region of VEGF and found that miR-93-5p may be proposed to reduce VEGF dependent angiogenesis in neuroblastomas [54]. Similarly, our results also predicted the putative miR-93-5p binding sites with 3'-UTR of VEGFa and luciferase reporter assay further confirmed that miR-95-5p directly targeted VEGFa. Additionally, a novel post-transcriptional regulatory mechanism of RNAs has been reported in which IncRNAs crosstalk with miRNAs through competing, functioning as competing endogenous RNAs and thus modulating the derepression of miRNAs on their targets [55]. For instance, Xiao et al. demonstrate that LncRNA MALAT1 may function as a competing endogenous RNAs to up-regulate Smad4 expression by sponging miR-204, promoting osteoblast differentiation of human aortic valve interstitial cells [28]. Furthermore, it has been found that LncRNA MEG3 directly targeting miR-133a-3p to inhibit the bone marrow mesenchymal stem cells differentiation in osteoporosis [56]. As shown in Fig. 7, in the present study, we also confirmed that AK131850 directly competed miR-93-5p in N-OC and M-OC through sponge, thus increasing VEGFa transcription, expression and secretion through derepressing of miR-93-5p on it. Moreover, the increased secretion of VEGFa in N-OC and M-OC remarkably promoted proliferation, differentiation, migration and tube formation of EPCs. In summary, we provided the first evidence that IncRNA-AK131850 sponged miR-93-5p in N-OC and M-OC to enhance the KARGER 
Quan et al.: LncRNA-AK131850 of Osteoclasts Enhances Vasculogenesis

secretion of VEGFa thus promoting vasculogenesis of EPCs.

Accordingly, we proposed an innovative view different from previous studies that not only PDGF-BB secreted by Pre-OC enhanced angiogenesis, but also VEGFa secreted by N-OC and $\mathrm{M}-\mathrm{OC}$ stimulated vasculogenesis during bone development and remodeling. These findings further confirmed that OCs at different stages during osteoclastogenesis all played a vital role in the processes of neovascularization pivotal to bone development and remodeling. Osteoclast-stimulated neovascularization was deserving of further investigation to more precisely define the molecular mechanism involved and to identify the role in physiological and pathological settings during bone development and remodeling. It may help develop novel therapeutic approaches for neovascularization in bone disorders such osteonecrosis.

\section{Acknowledgements}

This work was funded by the National Key Technology Research and Development Program of China (2017YFC1103300), the Nature Science Foundation of China (81572164), China Postdoctoral Science Foundation funded project (2016M592937) and Postdoctoral Research Project in Chongqing (Xm2016130).

\section{Disclosure Statement}

The authors report no potential conflicts of interests.

\section{References}

1 Huang B, Wang W, Li Q, Wang Z, Yan B, Zhang Z, Wang L, Huang M, Jia C, Lu J, Liu S, Chen H, Li M, Cai D, Jiang Y, Jin D, Bai X: Osteoblasts secrete Cxcl9 to regulate angiogenesis in bone. Nat Commun 2016;7:13885.

-2 Tomlinson RE, Silva MJ: Skeletal Blood Flow in Bone Repair and Maintenance. Bone Res 2013;1:311-322.

- Niedzwiedzki T, Filipowska J: Bone remodeling in the context of cellular and systemic regulation: the role of osteocytes and the nervous system. J Mol Endocrinol 2015;55:R23-36.

4 Carmeliet P, Jain RK: Molecular mechanisms and clinical applications of angiogenesis. Nature 2011;473:298-307.

5 Laschke MW, Giebels C, Menger MD: Vasculogenesis: a new piece of the endometriosis puzzle. Hum Reprod Update 2011;17:628-636.

6 Jing H, Liao L, Su X, Shuai Y, Zhang X, Deng Z, Jin Y: Declining histone acetyltransferase GCN5 represses BMSC-mediated angiogenesis during osteoporosis. FASEB J 2017;31:4422-4433.

7 Ramasamy SK, Kusumbe AP, Wang L, Adams RH: Endothelial Notch activity promotes angiogenesis and osteogenesis in bone. Nature 2014;507:376-380.

-8 Gotz W, Reichert C, Canullo L, Jager A, Heinemann F: Coupling of osteogenesis and angiogenesis in bone substitute healing - a brief overview. Ann Anat 2012;194:171-173.

-9 Wan C, Shao J, Gilbert SR, Riddle RC, Long F, Johnson RS, Schipani E, Clemens TL: Role of HIF-1alpha in skeletal development. Ann N Y Acad Sci 2010;1192:322-326.

10 Kusumbe AP, Ramasamy SK, Adams RH: Coupling of angiogenesis and osteogenesis by a specific vessel subtype in bone. Nature 2014;507:323-328.

11 Balaji S, King A, Crombleholme TM, Keswani SG: The Role of Endothelial Progenitor Cells in Postnatal Vasculogenesis: Implications for Therapeutic Neovascularization and Wound Healing. Adv Wound Care (New Rochelle) 2013;2:283-295.

12 Asahara T, Kawamoto A: Endothelial progenitor cells for postnatal vasculogenesis. Am J Physiol Cell Physiol 2004;287:C572-579.

13 Chen X, Zhi X, Cao L, Weng W, Pan P, Hu H, Liu C, Zhao Q Zhou Q, Cui J, Su J: Matrine derivate MASM uncovers a novel function for ribosomal protein S5 in osteoclastogenesis and postmenopausal osteoporosis. Cell Death Dis 2017;8:e3037. 


\section{Cellular Physiology Cell Physiol Biochem 2018;46:401-417 \begin{tabular}{l|l} 
DOI: 10.1159/000488474 & and Biochemistry \\
Published online: March 28, 2018 & $\begin{array}{l}\text { O 2018 The Author(s). Published by S. Karger AG, Basel } \\
\text { www.karger.com/cpb }\end{array}$
\end{tabular}}

Quan et al.: LncRNA-AK131850 of Osteoclasts Enhances Vasculogenesis

14 Percival CJ, Richtsmeier JT: Angiogenesis and intramembranous osteogenesis. Dev Dyn 2013;242:909-922.

15 Chen X, Zhi X, Pan P, Cui J, Cao L, Weng W, Zhou Q, Wang L, Zhai X, Zhao Q, Hu H, Huang B, Su J: Matrine prevents bone loss in ovariectomized mice by inhibiting RANKL-induced osteoclastogenesis. FASEB J 2017;31:4855-4865.

16 Dou C, Cao Z, Yang B, Ding N, Hou T, Luo F, Kang F, Li J, Yang X, Jiang H, Xiang J, Quan H, Xu J, Dong S: Changing expression profiles of IncRNAs, mRNAs, circRNAs and miRNAs during osteoclastogenesis. Sci Rep 2016;6:21499.

17 Bi H, Chen X, Gao S, Yu X, Xiao J, Zhang B, Liu X, Dai M: Key Triggers of Osteoclast-Related Diseases and Available Strategies for Targeted Therapies: A Review. Front Med (Lausanne) 2017;4:234.

18 Boyce BF: Advances in the regulation of osteoclasts and osteoclast functions. J Dent Res 2013;92:860-867.

19 Yoon CH, Hur J, Park KW, Kim JH, Lee CS, Oh IY, Kim TY, Cho HJ, Kang HJ, Chae IH, Yang HK, Oh BH, Park YB, Kim HS: Synergistic neovascularization by mixed transplantation of early endothelial progenitor cells and late outgrowth endothelial cells: the role of angiogenic cytokines and matrix metalloproteinases. Circulation 2005;112:1618-1627.

20 Gulati R, Simari RD: Cell therapy for angiogenesis: embracing diversity. Circulation 2005;112:1522-1524.

21 Hur J, Yoon CH, Kim HS, Choi JH, Kang HJ, Hwang KK, Oh BH, Lee MM, Park YB: Characterization of two types of endothelial progenitor cells and their different contributions to neovasculogenesis. Arterioscler Thromb Vasc Biol 2004;24:288-293.

22 Garimella R, Tague SE, Zhang J, Belibi F, Nahar N, Sun BH, Insogna K, Wang J, Anderson HC: Expression and synthesis of bone morphogenetic proteins by osteoclasts: a possible path to anabolic bone remodeling. J Histochem Cytochem 2008;56:569-577.

23 Yi T, Lee HL, Cha JH, Ko SI, Kim HJ, Shin HI, Woo KM, Ryoo HM, Kim GS, Baek JH: Epidermal growth factor receptor regulates osteoclast differentiation and survival through cross-talking with RANK signaling. J Cell Physiol 2008;217:409-422.

24 Xie H, Cui Z, Wang L, Xia Z, Hu Y, Xian L, Li C, Xie L, Crane J, Wan M, Zhen G, Bian Q, Yu B, Chang W, Qiu T, Pickarski M, Duong LT, Windle JJ, Luo X, Liao E, Cao X: PDGF-BB secreted by preosteoclasts induces angiogenesis during coupling with osteogenesis. Nat Med 2014;20:1270-1278.

25 Wang KC, Chang HY: Molecular mechanisms of long noncoding RNAs. Mol Cell 2011;43:904-914.

26 Klattenhoff CA, Scheuermann JC, Surface LE, Bradley RK, Fields PA, Steinhauser ML, Ding H, Butty VL, Torrey L, Haas S, Abo R, Tabebordbar M, Lee RT, Burge CB, Boyer LA: Braveheart, a long noncoding RNA required for cardiovascular lineage commitment. Cell 2013;152:570-583.

27 Tong X, Gu PC, Xu SZ, Lin XJ: Long non-coding RNA-DANCR in human circulating monocytes: a potential biomarker associated with postmenopausal osteoporosis. Biosci Biotechnol Biochem 2015;79:732-737.

28 Xiao X, Zhou T, Guo S, Guo C, Zhang Q, Dong N, Wang Y: LncRNA MALAT1 sponges miR-204 to promote osteoblast differentiation of human aortic valve interstitial cells through up-regulating Smad4. Int J Cardiol 2017;243:404-412.

29 Kwok ZH, Tay Y: Long noncoding RNAs: lincs between human health and disease. Biochem Soc Trans 2017;45:805-812.

30 Wapinski O, Chang HY: Long noncoding RNAs and human disease. Trends Cell Biol 2011;21:354-361.

-31 Whitehead J, Pandey GK, Kanduri C: Regulation of the mammalian epigenome by long noncoding RNAs. Biochim Biophys Acta 2009;1790:936-947.

32 Wang S, Tang C, Zhang Q Chen W: Reduced miR-9 and miR-181a expression down-regulates Bim concentration and promote osteoclasts survival. Int J Clin Exp Pathol 2014;7:2209-2218.

-33 Zhai Y, Tyagi SC, Tyagi N: Cross-talk of MicroRNA and hydrogen sulfide: A novel therapeutic approach for bone diseases. Biomed Pharmacother 2017;92:1073-1084.

34 Dou C, Zhang C, Kang F, Yang X, Jiang H, Bai Y, Xiang J, Xu J, Dong S: MiR-7b directly targets DC-STAMP causing suppression of NFATc1 and c-Fos signaling during osteoclast fusion and differentiation. Biochim Biophys Acta 2014;1839:1084-1096.

35 Wang Y, Kong D: Knockdown of IncRNA MEG3 inhibits viability, migration, and invasion and promotes apoptosis by sponging miR-127 in osteosarcoma cell. J Cell Biochem 2017;10.1002/jcb.26230

-36 Seemann I, Te Poele JA, Hoving S, Stewart FA: Mouse bone marrow-derived endothelial progenitor cells do not restore radiation-induced microvascular damage. ISRN Cardiol 2014;2014:506348. 


\section{Cellular Physiology Cell Physiol Biochem 2018;46:401-417

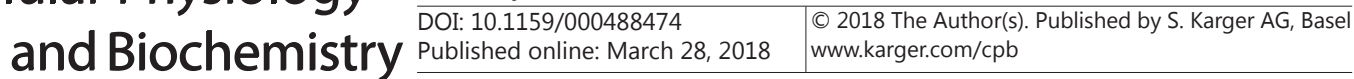

Quan et al.: LncRNA-AK131850 of Osteoclasts Enhances Vasculogenesis

-37 Yang NN, Jiao P, Li DW, Wang MZ, Yao ST, Zong CL, Qin SC: [Differential time attachment: optimization of the adherent time to obtain mouse bone marrow-derived endothelial progenitor cells]. Sheng Li Xue Bao 2011;63:574-580.

-38 Chen R, Yu H, Jia ZY, Yao QL, Teng GJ: Efficient nano iron particle-labeling and noninvasive MR imaging of mouse bone marrow-derived endothelial progenitor cells. Int J Nanomedicine 2011;6:511-519.

39 Ikeda K, Takeshita S: The role of osteoclast differentiation and function in skeletal homeostasis. J Biochem 2016;159:1-8.

-40 Kong L, Wang B, Yang X, Guo H, Zhang K, Zhu Z, Liu J, Hao D: Picrasidine I from Picrasma Quassioides Suppresses Osteoclastogenesis via Inhibition of RANKL Induced Signaling Pathways and Attenuation of ROS Production. Cell Physiol Biochem 2017;43:1425-1435.

-41 Ghosh A, Dasgupta D, Ghosh A, Roychoudhury S, Kumar D, Gorain M, Butti R, Datta S, Agarwal S, Gupta S, Dhali GK, Chowdhury A, Schmittgen TD, Kundu GC, Banerjee S: MiRNA199a-3p suppresses tumor growth, migration, invasion and angiogenesis in hepatocellular carcinoma by targeting VEGFA, VEGFR1, VEGFR2, HGF and MMP2. Cell Death \& Disease 2017;8:

42 Claesson-Welsh L, Welsh M: VEGFA and tumour angiogenesis. Journal of Internal Medicine 2013;273:114127.

43 Moya ML, Garfinkel MR, Liu X, Lucas S, Opara EC, Greisler HP, Brey EM: Fibroblast Growth Factor-1 (FGF-1) Loaded Microbeads Enhance Local Capillary Neovascularization. Journal of Surgical Research 2010;160:208-212.

44 Maynard SE, Min JY, Merchan J, Lim KH, Li JY, Mondal S, Libermann TA, Morgan LP, Sellke FW, Stillman IE, Epstein FH, Sukhatme VP, Karumanchi SA: Excess placental soluble fms-like tyrosine kinase 1 (sFlt1) may contribute to endothelial dysfimction, hypertension, and proteinuria in preeclampsia. Journal of Clinical Investigation 2003;111:649-658.

45 Guo Y, Wang J, Zhu M, Zeng R, Xu Z, Li G, Zuo B: Identification of MyoD-Responsive Transcripts Reveals a Novel Long Non-coding RNA (lncRNA-AK143003) that Negatively Regulates Myoblast Differentiation. Sci Rep 2017;7:2828.

46 Zhu L, Xu PC: Downregulated LncRNA-ANCR promotes osteoblast differentiation by targeting EZH2 and regulating Runx2 expression. Biochem Biophys Res Commun 2013;432:612-617.

47 Gerber HP, Vu TH, Ryan AM, Kowalski J, Werb Z, Ferrara N: VEGF couples hypertrophic cartilage remodeling, ossification and angiogenesis during endochondral bone formation. Nat Med 1999;5:623-628.

48 Ferrara N, Gerber HP, LeCouter J: The biology of VEGF and its receptors. Nat Med 2003;9:669-676.

49 Sakai G, Tokuda H, Fujita K, Kainuma S, Kawabata T, Matsushima-Nishiwaki R, Kozawa O, Otsuka T: Heat Shock Protein 70 Negatively Regulates TGF-beta-Stimulated VEGF Synthesis via p38 MAP Kinase in Osteoblasts. Cell Physiol Biochem 2017;44:1133-1145.

50 Grunewald M, Avraham I, Dor Y, Bachar-Lustig E, Itin A, Jung S, Chimenti S, Landsman L, Abramovitch R, Keshet E: VEGF-induced adult neovascularization: recruitment, retention, and role of accessory cells. Cell 2006;124:175-189.

-51 Lee SH, Jeong D, Han YS, Baek MJ: Pivotal role of vascular endothelial growth factor pathway in tumor angiogenesis. Ann Surg Treat Res 2015;89:1-8.

52 Koch S, Claesson-Welsh L: Signal transduction by vascular endothelial growth factor receptors. Cold Spring Harb Perspect Med 2012;2:a006502.

53 Koch S, Tugues S, Li X, Gualandi L, Claesson-Welsh L: Signal transduction by vascular endothelial growth factor receptors. Biochem J 2011;437:169-183.

54 Fabbri E, Montagner G, Bianchi N, Finotti A, Borgatti M, Lampronti I, Cabrini G, Gambari R: MicroRNA miR93-5p regulates expression of IL-8 and VEGF in neuroblastoma SK-N-AS cells. Oncol Rep 2016;35:28662872.

55 Tay Y, Rinn J, Pandolfi PP: The multilayered complexity of ceRNA crosstalk and competition. Nature 2014;505:344-352.

56 Wang Q, Li Y, Zhang Y, Ma L, Lin L, Meng J, Jiang L, Wang L, Zhou P, Zhang Y: LncRNA MEG3 inhibited osteogenic differentiation of bone marrow mesenchymal stem cells from postmenopausal osteoporosis by targeting miR-133a-3p. Biomed Pharmacother 2017;89:1178-1186. 